\title{
Distinction of Students and Expert Therapists Based on Therapeutic Motions on a Robotic Device Using Support Vector Machine
}

\author{
Koike Yuji ${ }^{1}$ (1) $\cdot$ Suzuki Makoto ${ }^{2} \cdot$ Okino Akihisa $^{3} \cdot$ Takeda Kazuhisa $^{3} \cdot$ Takanami Yasuhiro $^{4} \cdot$ Hamaguchi Toyohiro $^{1}$ (i)
}

Received: 26 February 2020 / Accepted: 25 August 2020 / Published online: 4 September 2020

(c) The Author(s) 2020

\begin{abstract}
Purpose To clarify the feature values of exercise therapy that can differentiate students and expert therapists and use this information as a reference for exercise therapy education.

Methods The participants were therapists with 5 or more years of clinical experience and 4th year students at occupational therapist training schools who had completed their clinical practices. The exercise therapy task included Samothrace (code name, SAMO) exercises implemented on the elbow joint based on the elbow flexion angle, angular velocity, and exercise interval recordings. For analyses and student/therapist comparisons, the peak flexion angle, peak velocity, and movement time were calculated using data on elbow angle changes acquired via SAMO. Subsequently, bootstrap data were generated to differentiate between the exercise therapy techniques adopted by therapists and students, and a support vector machine was used to generate four types of data combinations with the peak flexion angle, peak velocity, and movement time values. These data were used to estimate and compare the respective accuracies with the Friedman test.

Results The peak flexion angles were significantly smaller in the case of students. Furthermore, the peak velocities were larger, the peak flexion angles were smaller, and the movement times were shorter compared with those of therapists. The combination of peak velocity and peak flexion angle yielded the highest diagnostic accuracies.

Conclusion When students and therapists performed upper limb exercise therapy techniques based on the kinematics movement of a robot arm, the movement speeds and joint angles differed. The combination of peak velocity and peak flexion angle was the most effective classifier used for the differentiation of the abilities of students and therapists. The peak velocity and peak flexion angle of the therapist group can be used as a reference for students when they learn upper limb therapeutic exercise techniques.
\end{abstract}

Keywords Patient robot $\cdot$ Education $\cdot$ Spasticity $\cdot$ Rehabilitation $\cdot$ Occupational therapist

\section{Introduction}

Students who aim to become therapists study at rehabilitation training schools to acquire exercise therapy skills to master the relevant techniques. During their training, they

Hamaguchi Toyohiro

hamaguchi-toyohiro@spu.ac.jp

1 Department of Rehabilitation, Graduate School of Health Sciences, Saitama Prefectural University, Koshigaya, Saitama, Japan

2 Faculty of Health Sciences, Tokyo Kasei University, Sayama, Saitama, Japan

3 Okino Robotics Industries, Ltd, Kamikawa, Saitama, Japan

4 Peritec Corporation, Takasaki, Gunma, Japan learn manual exercise therapy theory from textbooks and study techniques using video materials. In addition, the students perform exercises on movement-impaired patients to acquire practical training, and work together to emulate the execution of the techniques/exercises. However, based on these study methods, the exercise therapy techniques learnt by students have been shown to be insufficient compared with practicing therapists [1]. As a result, students are not sufficiently skilled to carry out care independently, and technical skill training is thus needed [2]. The learning process of therapeutic exercise techniques is classified into three phases: (1) cognitive, (2) associative, and (3) autonomous phases $[3,4]$. The cognitive phase is the first step of the process in which students learn the types of exercises needed to acquire the required knowledge. At this stage, their movements are not sufficiently refined during the execution of 
the exercises, they lack accuracy, and they are slow and inefficient. The associative phase is the phase in which the student learning shifts from the concept on "what to do" to the concept "on how to do it," and the execution of the exercises gradually becomes increasingly accurate and efficient. In the autonomous phase, students are now able to execute exercises without focusing completely on what they do, and in this final phase they have eliminated unnecessary movements $[3,5]$. In other words, in their effort to learn and advance their skills, the student's capacity to master the therapeutic exercise technique remains at the cognitive phase level, and they encounter difficulties in reaching the next stage, that is, the associative phase. The lack of contact with the actual patients and their inability to practice the current technique-learning style on patients are some of the reasons for this. As a result, it is difficult for students to understand aspects of pathological patient movements, such as those associated with the stiffness of muscles and ligaments.

In addition, in recent years, medical education had been requested to (a) clarify the characteristics of necessary skills and (b) advance students education based on their individual abilities (outcome-based education) [6]. Accordingly, an important aspect of the evaluation of their education is related to whether sufficient knowledge and skills have been acquired pertaining to the manual execution of therapeutic exercise techniques. Nevertheless, there are no means available for teaching staff and instructors to determine the levels of skills acquired by students regarding exercise therapy techniques, such as the application of loads to patient joints and movement speed. Additionally, given that students rarely have the opportunity to work with real patients, the use of a simulated patient for objective structured clinical examination [7] is common. In other words, the teaching staff members are unable to verify whether the students have acquired sufficient knowledge/expertise for manually executing therapeutic exercise techniques.

Therefore, there is a need to develop new methods for teaching the execution of therapeutic exercise techniques and for determining the capacity level achieved by the student. Given that prior research has shown that the patient robots used in simulation education could be effective for training and technique evaluation $[8,9]$, this study developed an arm robot for this purpose (development code Samothrace: SAMO, patent no. 6307210) [1]. Given that the SAMO system can emulate patient pathology, it enables repeated practice in conditions that are similar to those required to treat an actual patient. In addition, the joint movements applied to the robot by human users can be recorded, and this data can be used to determine the proficiency with which the exercise therapy is being executed. However, to determine the achieved level of proficiency, it is important to clarify the feature values that students should aim for when they execute the therapeutic exercises. If an arm robot system like SAMO that recreates pathological movements of stroke patients can be used (a) as a tool for teaching therapeutic exercise techniques to students and (b) to present the differences between the techniques adopted by students and practicing therapists and the necessary feature values for treatment, this could facilitate the student's transition to the associative phase and solve problems encountered in the learning process. We hypothesize that therapists and students have different therapeutic exercise techniques, and the classifiers for distinguishing them are the range of motion, the velocity at which the therapeutic exercise techniques is performed, and the movement time. Accordingly, the goal of this study is clarification of the feature values to differentiate between the therapeutic exercise techniques adopted by students and therapists so they can be used as a reference for therapeutic exercise education.

\section{Subjects and Methods}

\subsection{Subjects}

The subjects were therapists with 5 or more years of clinical experience and 4th year students at occupational therapist training schools who had completed their clinical practices. The effect size of this study was estimated using the statistical software $G^{*}$ Power (a tool for computing statistical power analyses) based on available data, subject to the set levels of $\alpha=0.05$ and $1-\beta=0.80$ [10]. The minimum required number of participants was eight.

This study was approved by the Saitama Prefectural University Ethics Committee (approval no. 27112) and conducted in accordance with the Declaration of Helsinki. All participants received a thorough explanation of the experimental content in advance and gave their written consent for participation in the study.

\subsection{Therapeutic Exercise Results and Measurement Methods}

Students and therapists were asked to perform exercise therapies suited to the pathology settings of the SAMO system. Both groups were allowed to freely choose their postures, grip positions, and exercise therapy speeds (Fig. 1). With the SAMO elbow joint set to the stiffness mode, the subjects were asked to move the joint from maximum extension to maximum flexion, and then return it to maximum extension for two full cycles performed for four repetitions. The peak flexion angle, peak velocity, and movement time were calculated from the elbow angle changes recorded by SAMO. Data were recorded chronologically with a sampling rate of $100 \mathrm{~Hz}$. The elbow joint angle was calculated based on the length of wire wound by the actuator. The peak flexion 


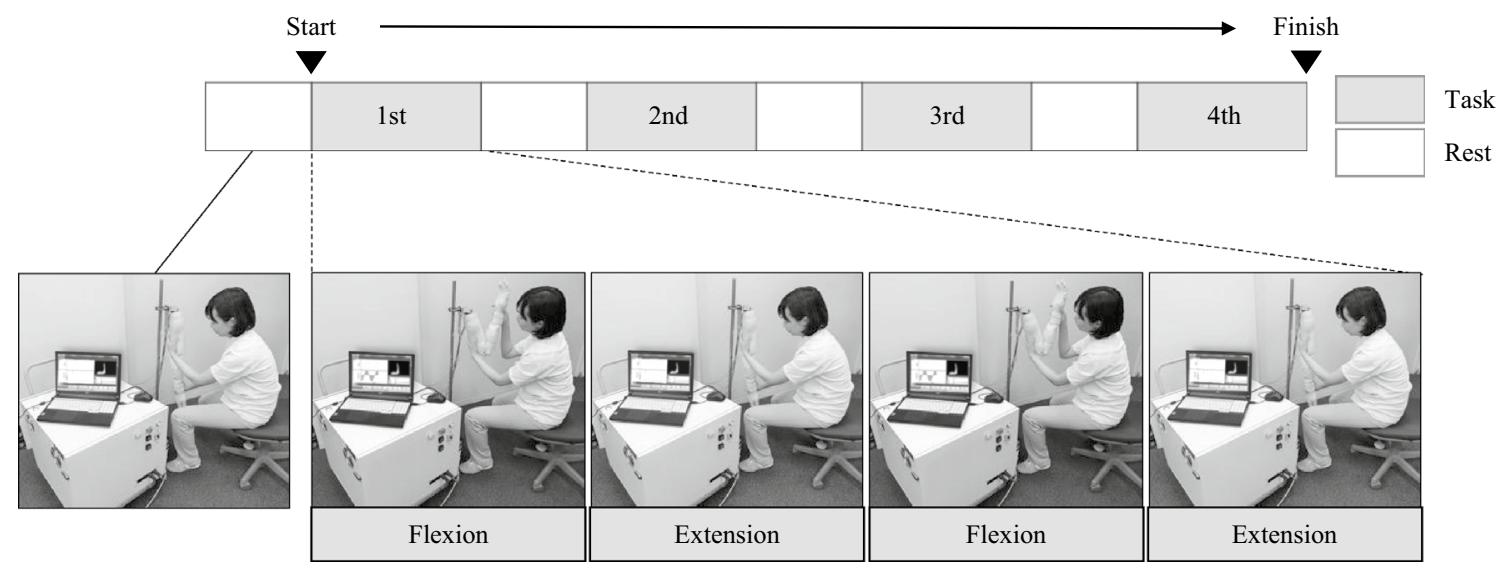

Fig. 1 Manual exercise therapy protocol. Gray and white boxes indicate the task and rest. The task involved the flexing of the elbow joint from the maximum extension position to the maximum flexion position, followed by its extension to the maximum extension position.

angle was calculated as the maximum value in the therapeutic exercise technique, and the full extension angle was calculated as the minimum value in the latter half of the therapeutic exercise technique. At the start of the movement time, the value detected exceeded the elbow's full extension angle on five consecutive time points. At the end of movement time, the value was defined at the point at which the elbow joint angle decreased to the full extension angle on five consecutive time points. The movement time was defined as the interval from the onset of the exercise to the end of the exercise. The angular velocity of the joint was obtained by dividing the angle by the time interval. There are four sets of exercises during an elbow flexion-extension exercise. Sets 1 to 3 were treated as practice runs, and only the data obtained at the 4th set of the exercise were used for analyses.

\subsection{Analyses}

The data from one full cycle of elbow exercise from maximum flexion to maximum extension and back to maximum flexion were used for analysis. The analysis items included the peak flexion angle, peak velocity, and movement time. These were estimated from the elbow angle changes recorded by SAMO during the exercises.

First, we compared the basic information of the subjects in this study, such as age, sex, and years after being registered. Second, to confirm that the 4th training data was stable, we compared the fluctuations of the 1st to 3rd training data obtained from the therapists and students. Third, to compare the differences between the therapists and students during the therapeutic exercise technique on a robot, we compared the 4th kinematics data from the therapists and students. To perform machine learning, we generated 2000
This was performed twice in a consecutive manner. The task was performed four times. The posture, gripping position, holding style, and task execution speed were unspecified, and the subjects were instructed to perform exercise therapy suited to the SAMO conditions

bootstrap data values (students $=1000$ and therapists $=1000$ ) by randomly drawing a series of sample data from the actual kinematics-related data of the 4 th. It has been reported that bootstrap data is stable at 1000 or more data items in the machine learning method based on the bootstrap method [11]; in this study, it was amplified to more than 2000 data items. The bootstrap resampling method has been extensively used in demographic studies [12-14]. Fourth, to confirm that the characteristics of the kinematics data are the same, we compared the bootstrap data from therapists and students.

The bootstrap kinematics-related data were then input into a nonlinear support vector machine (SVM) [15]. This powerful classification method can discriminate nonlinearly separable data using kernel functions to map the data to a higher dimensional space wherein the data become more separable $[15,16]$. In this study, the SVM focused on the peak flexion angle, peak velocity, and movement time patterns, and found the hypersurface that maximized the margin between the therapist and student's distributions to classify the data into the two groups. The bootstrap kinematics-related data were then randomly classified into 1500 training data values and 500 testing data values. An SVM algorithm was then formulated based on the training dataset to establish the classification model. Fifth, to verify the combinations of kinematics-related data used for the SVM classifier that can be discriminated most correctly, we determined the combinations of four kinematics-related data as (1) the peak velocity, peak flexion angle, and movement time, (2) the peak velocity and peak flexion angle, (3) the peak velocity and movement time, and (4) the peak flexion angle and movement time. After training, the SVM classification model was applied to the training dataset to assign the testing dataset to the appropriate therapists. This validation 
procedure was repeated 10 times, and the accuracy rate was calculated as $\mathrm{AR}=(\mathrm{TP}+\mathrm{TN}) / \mathrm{N}$, where $\mathrm{AR}$ is the accuracy rate, TP is the true positive, TN is the true negative, and $\mathrm{N}$ is the sum of true positive, true negative, false positive, and false negative. This ensured that the trained SVM can generalize the data such that they could be applied to the peak flexion angle, peak velocity, and movement time patterns when they are presented to the SVM algorithm [17].

In the statistical analysis, first, we tested the normality of the kinematics data before amplification using the bootstrap method. No normality was found; therefore, the Mann-Whitney U test was performed to compare the therapists and students' therapeutic exercise technique. The Mann-Whitney U test was also used to compare the bootstrap data obtained from therapists and students. Friedman tests were performed to compare the 1 st to 3 rd data within each group and the accuracy rates. If a significant difference is found, the Steel-Dwass test was performed as a post-hoc test. The analyses were performed using with the R 3.4.0 software (R Foundation for Statistical Computing, Vienna, Austria).

\section{Results}

The participants of this study were 11 therapists with 5 or more years of clinical experience ( 1 female, 10 male) and 11 4th year students at occupational therapist training schools

Table 1 Participant characteristics

\begin{tabular}{lll}
\hline & $\begin{array}{l}\text { Therapists } \\
(\mathrm{n}=11)\end{array}$ & $\begin{array}{l}\text { Students } \\
(\mathrm{n}=11)\end{array}$ \\
\hline Age (years) & $35.0(32.0-37.0)$ & $22.0(22.0-22.0)$ \\
Sex (male/female) & $10 / 1$ & $2 / 9$ \\
Years after registered & $12.0(9.0-15.0)$ & 0 \\
\hline
\end{tabular}

Numbers are means $(25-75 \%)$ who had completed their clinical practices (nine females, two males) (Table 1). The median clinical experiences of the therapists after they had acquired their licenses were 12.0 years.

\subsection{Comparison of the Exercise Therapy Technique of Therapists and Students}

The kinematics-related data for the SAMO exercise therapies performed by the therapists and students (elbow joint angle, angular velocity, and movement time) were compared, and variations in the data from practice runs $1-3$ for the therapist and student groups are presented in Table 2. There were no significant differences within the practice run data for either group. A scatter plot of the kinematics-related data for the 4th repetition of the exercise therapy task (therapist and student groups) is shown in Fig. 2. When the data for the therapists and students were compared, there were no significant differences in the peak velocity and movement time between the groups. There was a small but significant difference in the peak flexion angle data $(p=0.002, r=0.67)$ (Table 3).

\subsection{Therapist and Student Exercise Therapy Technique Classifiers}

To differentiate between the kinematics-related data for the therapists and students using SVM, the actual data for the peak velocity, peak flexion angle, and movement time were amplified using the bootstrap method (generated data) (Fig. 3). When these generated data were used to compare the exercise therapy technique of therapists and students, we found that the students' peak velocity was significantly greater $(p<0.001, r=1.26)$, the peak flexion angle was smaller $(p<0.001, r=8.23)$, and the movement time was shorter $(p<0.001, r=4.15)$ compared with those of the therapists (Table 4).

Table 2 Differences in kinematics-related data within group (therapists and students) according to manual therapy trials

\begin{tabular}{|c|c|c|c|c|c|c|}
\hline & 1st trial & 2nd trial & 3rd trial & $\chi^{2}$ & $d f$ & $p$ value \\
\hline \multicolumn{7}{|l|}{ Therapist } \\
\hline Peak velocity $(\% / s)$ & $108.2(79.5-144.1)$ & $137.3(93.2-161.4)$ & $135.5(108.2-161.4)$ & 2.930 & 2 & 0.231 \\
\hline Peak flexion angle $\left({ }^{\circ}\right)$ & $119.5(116.9-125.5)$ & $120.4(116.4-125.0)$ & $119.7(117.4-122.4)$ & .727 & 2 & 0.695 \\
\hline Movement time (s) & $10.8(7.3-14.4)$ & $9.2(7.3-12.6)$ & $8.3(7.6-11.9)$ & 1.273 & 2 & 0.529 \\
\hline \multicolumn{7}{|l|}{ Students } \\
\hline Peak velocity $(\% / s)$ & $110.0(98.6-169.6)$ & $124.6(76.8-143.6)$ & $141.8(100.5-154.1)$ & 3.395 & 2 & 0.183 \\
\hline Peak flexion angle $\left({ }^{\circ}\right)$ & $111.5(98.0-112.0)$ & $110.9(100.0-114.2)$ & $112.5(98.0-115.4)$ & 5.091 & 2 & 0.078 \\
\hline Movement time (s) & $7.9(5.5-13.5)$ & $8.5(7.0-13.4)$ & $9.1(6.4-12.4)$ & 2.909 & 2 & 0.234 \\
\hline
\end{tabular}

Numbers are means $(25-75 \%)$

Friedman test for therapists $(\mathrm{n}=11)$ and students $(\mathrm{n}=11)$ 

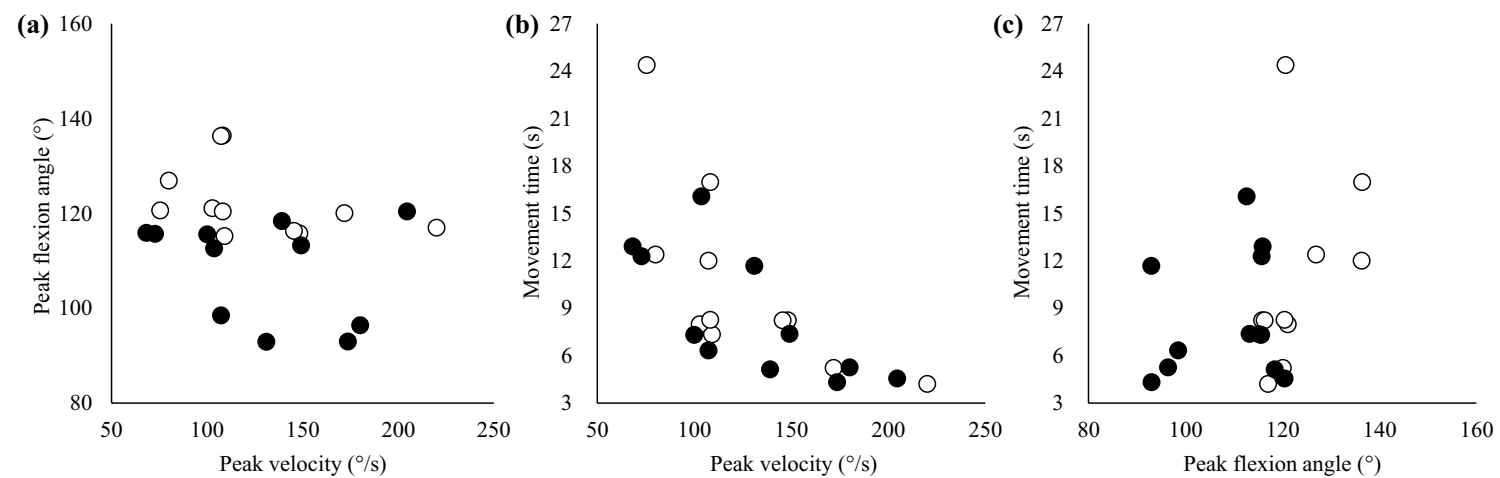

Fig. 2 Scatter plot of actual kinematics data during manually executed exercise therapy. a Peak angle and peak velocity, b movement time and peak velocity, c movement time and peak angle during

manually executed therapy on a robot arm. White circle: therapists $(n=11)$, black circle: students $(n=11)$
Table 3 Comparisons of actual kinematics data between therapists and students

\begin{tabular}{lcclll}
\hline & Therapists $(\mathrm{n}=11)$ & Students $(\mathrm{n}=11)$ & Z value & p value & $r$ \\
\hline Peak velocity $(\% / \mathrm{s})$ & $108.2(105.0-146.8)$ & $130.9(101.8-161.4)$ & 0.66 & 0.948 & 0.14 \\
Peak flexion angle $\left({ }^{\circ}\right)$ & $120.4(116.7-124.0)$ & $113.2(97.5-115.8)$ & 3.119 & $0.002^{*}$ & 0.67 \\
Movement time $(\mathrm{s})$ & $8.2(7.7-12.2)$ & $7.3(5.2-12.0)$ & 1.018 & 0.309 & 0.22 \\
\hline
\end{tabular}

Numbers are means $(25-75 \%)$

$* p<0.01$

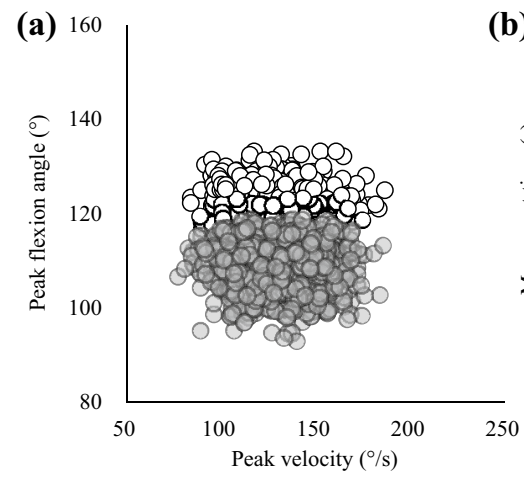

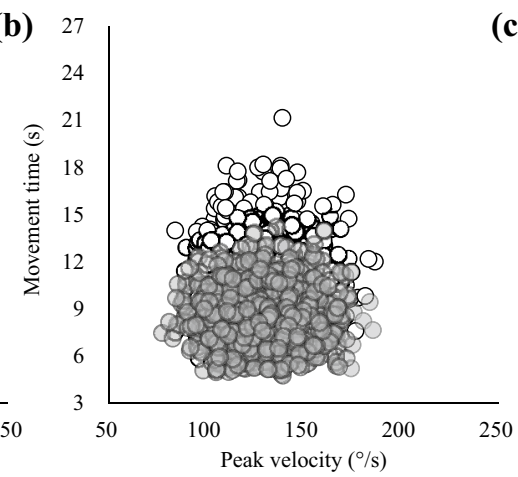

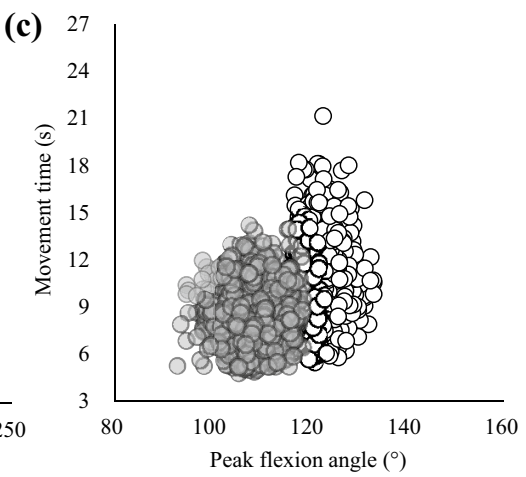

Fig. 3 Scatter plot of bootstrap kinematics data during manually executed exercise therapy. a Peak angle and peak velocity. b Movement time and peak velocity. $\mathbf{c}$ Movement time and peak angle during manually executed therapy on a robot arm. White circle: therapists $(n=1000)$, transparent gray circle: students $(n=1000)$
Table 4 Comparisons of generated kinematics data between therapists and students

\begin{tabular}{lccrrr}
\hline & Therapists $(\mathrm{n}=11)$ & Students $(\mathrm{n}=11)$ & Zvalue & p value & $r$ \\
\hline Peak velocity $(\% / \mathrm{s})$ & $123.6(110.9-136.7)$ & $130.0(116.9-142.2)$ & 5.889 & $<0.001^{*}$ & 1.26 \\
Peak flexion angle $\left({ }^{\circ}\right)$ & $122.0(120.0-125.0)$ & $108.6(105.5-112.1)$ & 38.617 & $<0.001^{*}$ & 8.23 \\
Movement time $(\mathrm{s})$ & $10.4(8.7-12.3)$ & $8.3(7.3-9.6)$ & 19.473 & $<0.001^{*}$ & 4.15 \\
\hline
\end{tabular}

Numbers are means $(25-75 \%)$

$*_{p}<0.01$ 


\subsection{Use of Combinations of Exercise Variables to Differentiate the Exercise Therapy Technique of Therapists and Students}

The bootstrap data (amplified to 1000 times) was used to create four different combinations of kinematics-related data of students and therapists. In total, 1500 samples were defined as training data for SVM learning, and the classifiers were established. The remaining 500 samples were used with these classifiers to automatically differentiate the therapists and students' techniques. The best combination of characteristics was determined (Fig. 4). The success rates for the four combinations were as follows: peak velocity and peak flexion angle: $96.4 \pm 0.0 \%$, peak flexion angle and movement time: $86.1 \pm 0.0 \%$, peak velocity, peak flexion angle, and movement time: $61.5 \pm 0.0 \%$, and peak velocity and movement time: $56.4 \pm 0.0 \%$. There were significant differences in the accuracy of the four kinematics-related data combinations (chi-squared $=29.3$, degrees of freedom $=9, p=0.001$, $\left.\eta^{2}=0.98\right)$. The results of a post-hoc test showed that the combination of peak velocity and peak flexion angle yielded the highest accuracy $(t=3.8, p=0.001$, $d=6.33-25.30$ ).

\section{Discussion}

This study used SVM to generate bootstrap kinematicsrelated data, determine the characteristics of the actual kinematics data, and verify the capacity to distinguish the therapeutic exercise techniques adopted by students and therapists. The results showed that the combination of peak velocity and peak flexion angle was the most accurate feature value classifier. In addition, there were kinematics differences in the peak velocity, peak flexion angle, and movement time between the two groups. In this study, the therapeutic exercise technique of students was different from that of therapists. Our results also support the hypothesis that the therapeutic exercise technique of students and therapists can

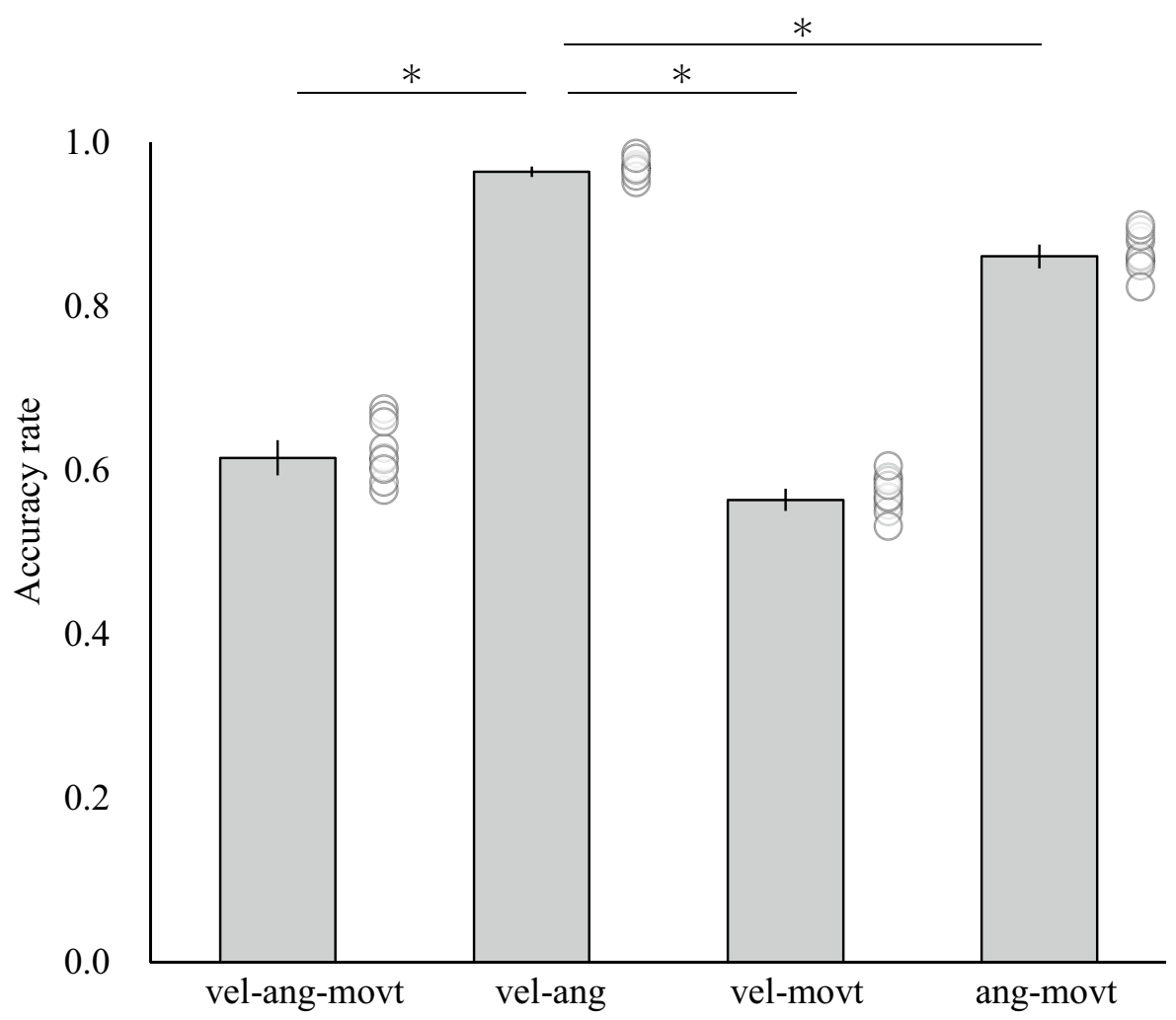

Fig. 4 Comparison of accuracy rate by support vector machine (SVM) trained kinematics data during manually executed exercise therapy. Significant differences were found by the Friedman rank sum test (chi-squared $=29.3$, degrees of freedom $=9, p=.001, \eta^{2}=.98$ ). Significant differences were found by the post-hoc Steel-Dwass test. The accuracy rate of vel-ang was significantly higher than that for ang-movt $(\mathrm{t}=3.8, p=.001, d=6.33)$, vel-ang-movt $(t=3.8, p=.001$, $d=15.21)$, and vel-movt $(t=3.8, p=.001, d=25.30)$. The accuracy rate of ang-movt was significantly higher than vel-ang-movt $(t=3.8$, $p=.0009, d=9.41)$ and vel-movt $(t=3.8, p=.001, d=15.00)$. The accuracy rate of vel-ang-movt was higher than vel-movt $(t=3.2$, $p=.007, d=2.35$ ). White circles are the accuracy rates estimated based on 10 iterations. Bars indicate means and standard deviations of accuracy rate, and white circles are estimated accuracy rates for 10 trials (vel: peak velocity, ang: peak flexion angle, movt: movement time). ${ }^{*} p<.001$ 
be discriminated by the classifier of SVM with kinematics data as a feature quantity. These differences indicate that the students moved the patient arm faster, did not achieve the maximum possible angle of movement, and spent less time on the exercise therapies. The therapists were able to bend slowly up to a maximum angle. It is considered that therapists were careful in their movements because they have clinically treated patients. In other words, when carrying out the therapeutic exercise technique, the muscle tone is speed-dependent [18], and the knowledge that a fast movement causes stretch reflex to occur and that exercise is performed to the maximum angle is not limited to therapists. However, the students lacked specific knowledge such as how fast they actually move to cause stretch reflexes and increase muscle tone, and resistance in the final region to judge the maximum angle. On the other hand, it is considered that therapists who carry out the therapeutic exercise technique every day have knowledge about the exercise and can practice. Students learned these therapeutic exercise techniques from textbooks, video learning materials, emulation practices with other students, and short-term clinical practice. In exercise therapy, the optimal movement speed and range for the patients' upper limbs were determined by their pathologies. Students had trouble recognizing the motor components of the therapeutic exercise technique and learning them proved difficult. The results of this study suggests that the movement speed and angle of the patient's arm are motion components that can be learned by students based on reference data from therapists.

Machine learning research studies have reported that the potential of the active lower limb muscles during walking can be classified as motion indicators using SVM [19]. In addition, a machine learning differentiation method has been proposed using vector quantization (VQ) [20]. The differentiation of the exercise therapy techniques adopted by therapists and students performed in this study could also employ VQ. However, the feature values of kinematics-related data are unknown in VQ. For this reason, even if the distinction of the therapeutic exercise technique adopted by therapists and students is possible, this does not constitute a conclusive comparison of the two groups and does not clarify the goal values. The limitation of this method is that identifying the motion components that are problematic for student learning is difficult. Accordingly, SVM is advantageous because it can clarify the factor values, such as the kinematics angles and speed, that differ between the two groups.

In this study, SVM was used to distinguish the therapeutic exercise techniques adopted by students and therapists. To increase the accuracy of SVM classifiers, a large volume of training data is required. The investigation environment consisted of one SAMO unit, the number of student and therapist subjects was small, and the accumulation of sufficient, actual data for use as training data was difficult.
For these reasons, the study used the bootstrap method to amplify the actual data and generate training data. The data values generated using the bootstrap method was within the measurement error range, and had a smaller standard error than the actual data. Accordingly, the risk rates were smaller, the differentiation boundaries were narrower, and differentiation failure could occur more easily. Compared with generated data, the standard error of large volumes of actual data is larger. This increases the SVM classifier accuracy. Thus, future studies should either increase the volume of actual data or widen the error range for the amplified data of the bootstrap method to create new classifiers.

Since the effect of stretch reflex on spasticity is speeddependent [18], the patient's muscle resistance increases when trying to move fast. Furthermore, the stronger the muscle tone, the greater the force required to administer therapeutic exercise technique [21]. Therefore, the velocity and angle elements as well as the force element are important for the therapeutic exercise technique. In other words, when teaching the therapeutic exercise technique to students, it is necessary to teach from the above viewpoint; however, this study could not evaluate the force. Therefore, SAMO should be updated so that the force applied to the arm can be analyzed.

\section{Conclusion}

The results of this study showed that the movement speed and angle of a kinematics robot arm differed when students and therapists performed therapeutic exercise techniques. In addition, the combination of the peak velocity and peak flexion angle was the most accurate classifier for the differentiation of the proficiencies of students and therapists. Based on these findings, the peak velocity and peak flexion angle achieved by therapists could be used as a reference by students in their efforts to learn the therapeutic exercise techniques of the arms.

Author Contributions KY and HT designed the study and wrote the initial draft of the manuscript. SM, OA, TK, and TY contributed to analysis and interpretation of data and assisted in the preparation of the manuscript. All other authors contributed to data collection and interpretation, and critically reviewed the manuscript. All authors approved the final version of the manuscript and agree to be accountable for all aspects of the work, ensuring that questions related to the accuracy or integrity of any part of the work are appropriately investigated and resolved.

Funding This work was supported by the Grant-in-Aid for Leadingedge Industry Design Project powered by the Saitama Prefecture 
2015-2017 to Hamaguchi Toyohiro, and Grant-in-Aid JSPS KAKENHI 17K13059 and 20K11286 to Koike Yuji.

Data Availability Subject data, except for personal information, will be provided in excel files upon researcher's request with the approval of the Ethics Committee. If a researcher wants to use the data, request should be made by e-mail to the corresponding author.

Code Availability The SAMO application was written in LabVIEW. This app is a product of Peritec Inc.

\section{Compliance with Ethical Standards}

Conflict of interest All authors declare that they have no conflict of interest.

Ethical Approval This study was approved by the Saitama Prefectural University Ethics Committee (Approval No. 27112).

Informed Consent This study was conducted in accordance with the Declaration of Helsinki. All participants received a thorough explanation of the experimental content in advance and gave their written consent for participation in the study.

Consent for Publication The Author warrants and represents that the Contribution does not infringe upon any copyright or other rights, and that it does not contain infringing or other unlawful matter, that he/ she was the sole and exclusive owner of the rights herein conveyed to the Publisher.

Open Access This article is licensed under a Creative Commons Attribution 4.0 International License, which permits use, sharing, adaptation, distribution and reproduction in any medium or format, as long as you give appropriate credit to the original author(s) and the source, provide a link to the Creative Commons licence, and indicate if changes were made. The images or other third party material in this article are included in the article's Creative Commons licence, unless indicated otherwise in a credit line to the material. If material is not included in the article's Creative Commons licence and your intended use is not permitted by statutory regulation or exceeds the permitted use, you will need to obtain permission directly from the copyright holder. To view a copy of this licence, visit http://creativecommons.org/licenses/by/4.0/.

\section{References}

1. Koike, Y., Suzuki, M., Okino, A., Takeda, K., Takanami, Y., \& Hamaguchi, T. (2019). Differences in manual exercise therapy skills between students and therapists. Journal of Ergonomic Technology, 19, 35-44.

2. Hodgetts, S., Hollis, V., Triska, O., Dennis, S., Madill, H., \& Taylor, E. (2007). Occupational therapy students' and graduates' satisfaction with professional education and preparedness for practice. Canadian Journal of Occupational Therapy, 74, 148-160.

3. Fitts, P. M. (1964). Perceptual-motor skill learning. In A. W. Melton (Ed.), Categories of human learning (pp. 243-285). New York: Academic Press.

4. Fitts, P. M., \& Posner, M. I. (1967). Human performance. Belmont: Wadsworth.
5. Schmidt, R. A., \& Wrisberg, C. A. (2008). Motor learning and performance: A situation-based learning approach. Champaign, IL: Human Kinetics.

6. Harden, R. M. (2002). Learning outcomes and instructional objectives: is there a difference? Medical Teaching, 24, 151-155.

7. Harden, R. M. (1988). What is an OSCE? Medical Teaching, 10, 19-22.

8. Tanzawa, T., Futaki, K., Kurabayashi, H., Goto, K., Yoshihama, Y., Hasegawa, T., et al. (2013). Medical emergency education using a robot patient in a dental setting. European Journal of Dental Education, 17, e114-e119.

9. Takanobu, H., \& Bozovic, V. (2008). Dental patient robot: Medical robotics. In V. Bozovic (Ed.), Medical robotics (Chapter 32, pp. 443-456). Shanghai: I-Tech Education and Publishing.

10. Faul, F., Erdfelder, E., Lang, A. G., \& Buchner, A. (2007). G*Power 3: A flexible statistical power analysis program for the social, behavioral, and biomedical sciences. Behavioral Research Methods, 39(2), 175-191.

11. Hedges, S. B. (1992). The number of replications needed for accurate estimation of the bootstrap p value in phylogenetic studies. Molecular Biology and Evolution, 9, 366-369.

12. Kim, S. H., Park, E. Y., Joo, J., \& Chung, J. (2018). The De Ritis and neutrophil-to-lymphocyte ratios may aid in the risk assessment of patients with metastatic renal cell carcinoma. Journal of Oncology, 2018, 1953571.

13. Chiu, C. T., Hayward, M. D., Chan, A., \& Matchar, D. B. (2019). Educational differences in the compression of disability incidence in the United States. SSM Population Health, 7, 100347.

14. Schaffar, R., Belot, A., Rachet, B., \& Woods, L. (2019). On the use of flexible excess hazard regression models for describing long-term breast cancer survival: A case-study using populationbased cancer registry data. BMC Cancer, 9(1), 107.

15. Davatzikos, C., Ruparel, K., Fan, Y., Shen, D. G., Acharyya, M., Loughead, J. W., et al. (2005). Classifying spatial patterns of brain activity with machine learning methods: Application to lie detection. Neuroimage, 28(3), 663-668.

16. Mohammadpoory, Z., Nasrolahzadeh, M., \& Haddadnia, J. (2017). Epileptic seizure detection in EEGs signals based on the weighted visibility graph entropy. Seizure, 50, 202-208.

17. Kloppel, S., Stonnington, C. M., Chu, C., Draganski, B., Scahill, R. I., Rohrer, J. D., et al. (2008). Automatic classification of MR scans in Alzheimer's disease. Brain, 131(Pt 3), 681-689.

18. Lance, J. W. (1980). Symposium synopsis. In R. Feldman, R. Young, \& W. Koells (Eds.), Spasticity: Disordered motor control (pp. 487-489). Chicago: Year Book Medical Publishers.

19. Ceseracciu, E., Reggiani, M., Sawacha, Z., Sartori, M., Spolaor, F., Cobelli, C., \& Pagello, E. (2010). SVM classification of locomotion modes using surface electromyography for applications in rehabilitation robotics. 19th IEEE International Symposium on Robot and Human Interactive Communication, 12-15.

20. Gray, R. M. (1984). Vector quantization. IEEE ASSP Magazine, 1, 4-29.

21. Pandyanm, A. D., Price, C. I. M., Rodgers, H., Barnes, M. P., \& Johnson, G. R. (2001). Biomechanical examination of a commonly used measure of spasticity. Clinical Biomechanics, 16, 859-865.

Publisher's Note Springer Nature remains neutral with regard to jurisdictional claims in published maps and institutional affiliations. 